\title{
Semiclassical approach to bound states of a pointlike impurity in a two-dimensional Dirac system
}

\author{
Kun Woo Kim, ${ }^{1}$ Tami Pereg-Barnea, ${ }^{2}$ and Gil Refael ${ }^{1}$ \\ ${ }^{1}$ Department of Physics, California Institute of Technology, 1200 East California Boulevard, MC114-36, Pasadena, CA 91125, USA \\ ${ }^{2}$ Department of Physics and Centre for Physics of Materials, McGill University, Montreal, Quebec, Canada H3A 2T8 \\ (Received 5 August 2013; revised manuscript received 5 January 2014; published 18 February 2014)
}

\begin{abstract}
The goal of this paper is to provide an intuitive and useful tool for analyzing the impurity-bound-state problem. We develop a semiclassical approach and apply it to an impurity in two-dimensional systems with parabolic or Dirac-like bands. Our method consists of reducing a higher-dimensional problem into a sum of one-dimensional ones using the two-dimensional Green's functions as a guide. We then analyze the one-dimensional effective systems in the spirit of the wave-function-matching method as in the standard one-dimensional quantum model. We demonstrate our method on two-dimensional models with parabolic and Dirac-like dispersion, with the later specifically relevant to topological insulators.
\end{abstract}

DOI: 10.1103/PhysRevB.89.085117

PACS number(s): 71.55.Jv, 03.65.Ge, 03.65.Sq, 03.65.Vf

\section{INTRODUCTION}

The presence of disorder is often considered a nuisance that degrades the quality of samples and obscures the behavior of clean physical systems. Even in small amounts, however, impurities may induce new phases which are interesting in their own right and do not have a clean-system analog. Prominent examples are the metal-insulator transition induced by random on-site potential [1-4], the Cooper-pair-breaking transition in conventional $s$-wave superconductors by magnetic [5-7] and nonmagnetic [8-10] impurities, the impurity-induced spin quantum Hall effect [11-13], and the Kondo effect [14,15].

Understanding the single-impurity problem often provides strong intuition for the behavior of a disordered system with a finite impurity density. Using this as motivation, we study the problem of bound states of a single narrow impurity in a variety of host systems. For a narrow impurity, bound states could be found most straightforwardly by solving the Schrödinger equation outside and inside the impurity-affected region and matching the wave functions at the boundary. In one dimension, with a $\delta$-function impurity potential, this is particularly simple. It is also quite straightforward when dealing with two-dimensional systems (2D) and a one-dimensional (1D) perturbation such as an edge. However, for pointlike impurities in two dimensions, a more complicated consideration is required.

Nevertheless, in this work we show that a 2D system with a pointlike impurity could be reduced to a $1 \mathrm{D}$ problem on a straight trajectory in which wave-function matching can be applied (Fig. 1). Furthermore, in spatially anisotropic systems (namely, lacking rotational symmetry about the impurity), we show that using a small number of incoming and outgoing beams, straight trajectories allow a remarkably accurate estimate of bound-state energies.

The semiclassical approach has been instrumental in providing insightful physical pictures in terms of classical trajectories in complicated quantum systems, especially when impurity scatterers or confining potentials are involved. Examples of such applications include quasiparticle states near extended scatterers in $d$-wave superconductors [16], bound states in multidimensional systems with Fermi resonance [17], the low-energy spectrum of charge carriers in graphene [18], and the Berry phase in graphene [19]. The standard semiclassical methods which map complicated multidimensional quantum problems onto a 1D quantum problem on simple classical trajectories, however, are approximations and suffer from limitations that need to be addressed with more sophisticated methods $[18,20]$.

In the following sections we derive the mapping from two dimensions to an effective 1D impurity Hamiltonian and then use it to find the bound-state energies in several examples of increasing complexity. This mapping should be thought of as a semiclassical description of the 2D impurity problem, where the bound-state energies are obtained by considering a small number of classical incoming and outgoing beams. The mapping from 2D to $1 \mathrm{D}$ relies on the Green's function of the clean system, which indicates which "classical" paths are necessary. Our method also approximates the bulk Hamiltonian by its form in the vicinity of minima in momentum space, assuming a parabolic or Dirac-like dispersion. We find that with the introduction of appropriate cutoffs using the Pauli-Villars regulators, this approximation remains relatively precise.

The organization of this paper is as follows. In Sec. II we show how the $2 \mathrm{D}$ impurity problem can be reduced to a $1 \mathrm{D}$ problem for an isotropic Hamiltonian system. In Sec. III, the method is extended to an anisotropic Hamiltonian, and then in Sec. IV it is extended to a band structure with multiple minima. While the extension of our method to any odd dimension is straightforward, the extension to even dimensions is not. In Sec. V we show how to generalize our method to all evendimensional systems.

\section{WAVE-MATCHING FOR A SINGLE ISOTROPIC MINIMUM CONTINUUM BAND}

\section{A. Review of the one-dimensional problem}

Our goal in this section is to derive a method equivalent to the 1D wave-function-matching technique to find the bound states of an impurity in an isotropic 2D system. For this purpose, let us briefly review how a bound state associated with a single point impurity is obtained in a $1 \mathrm{D}$ system. The Schrödinger equation in this case is simply

$$
\left[E-H\left(\partial_{x}\right)\right] \psi(x)=\alpha \delta(x) \psi(x),
$$


(a)

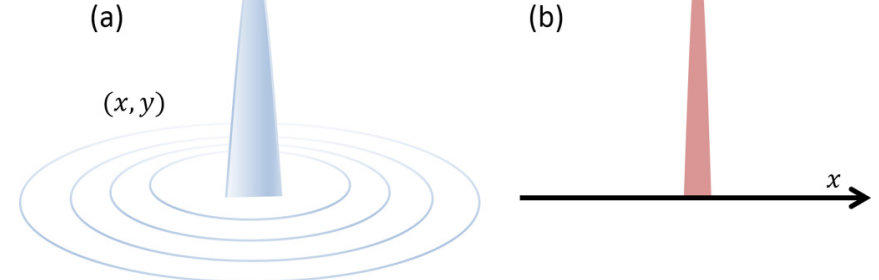

FIG. 1. (Color online) (a) A 2D system with a pointlike impurity is reduced to (b) the $1 \mathrm{D}$ system with a modified impurity strength. Thus, a simple picture of wave-function matching can be applied to $2 \mathrm{D}$ system to obtain a bound-state energy associated with a single impurity in two dimensions.

where the Hamiltonian $H$ is responsible for the kinetic part only and the impurity scattering strength is $\alpha$. The Green's function of the operator $E-H\left(\partial_{x}\right)$ is given by

$$
G(x)=\int_{-\infty}^{\infty} \frac{d k}{2 \pi} \frac{e^{i k x}}{E-\tilde{H}(k)},
$$

where $\tilde{H}(k)$ is the Hamiltonian in the momentum representation. The Green's function $G(x)$ is understood as the amplitude of the propagator at $x$, originating from the source at $x=0$. Equation (2) can be computed using contour integration around the upper (lower) half of a complex $k$ plane for positive (negative) $x$. If the energy $E$ is within the band, the (real-axis) poles of the integrand each correspond to a plane wave, and the combination of these waves makes up the Green's function. If the energy is outside the band, as would be the case for a bound state, the poles are not on the real axis, and therefore the wave function decays exponentially with distance. We can still think of such a Green's function as a combination of plane waves, but with complex wave vectors.

To obtain the bound-state energy associated with the impurity, we could take two paths. Formally, we use the fact that the Green's function is the solution of Eq. (1) omitting $\alpha \psi(x)$ on the right side of the equality. Therefore

$$
\psi(x)=G(x) \alpha \psi(0),
$$

and we obtain for a scalar Hamiltonian

$$
\frac{1}{\alpha}=G(0)=\int_{-\infty}^{\infty} \frac{d k}{2 \pi} \frac{1}{E-\tilde{H}(k)},
$$

which is consistent with the $T$-matrix formulation [21].

For a Hamiltonian with an internal structure such as sublattice or spin, the Green's function and the impurity potential are matrices. The impurity potential matrix $\alpha$ may not be invertible. In this case, for $\vec{\psi}(0)$ to have a nontrivial solution at $x=0$, the following condition is required:

$$
\operatorname{Det}[I-G(0) \alpha]=0 .
$$

For simplicity of presentation, in most of this paper we consider only scalar problems. In Sec. IV, along with extending our method to the case of host systems with multiple low-gap valleys, we also assume a multicomponent wave function.

An alternative to the above method is to solve the 1D equation [Eq. (1)] simply by matching a freely propagating plane-wave solution at $x>0$ with a different plane-wave solution at $x<0$. Integrating the Schrödinger equation over the impurity position gives

$$
\alpha \psi(0)-\int_{-\epsilon}^{\epsilon}(E-H) \psi(x) d x=0 .
$$

Indeed, the same bound energy relation as in Eq. (4) or Eq. (5) is obtained from this wave-function-matching approach by inserting Eq. (3) in the above equation.

\section{B. Effective one-dimensional problem}

Could we use the same notion of wave-function matching in the context of a bound state in two dimensions? Let us start with the 2D Schrödinger equation:

$$
\left[E-H_{2 d}(\vec{r})\right] \Psi(\vec{r})=\alpha \delta^{2}(\vec{r}) \Psi(\vec{r}) .
$$

To solve for a bound state at energy $E$, we can still use Eq. (3), adapted to two dimensions with

$$
G(x)=\int_{-\infty}^{\infty} \frac{d^{2} k}{(2 \pi)^{2}} \frac{e^{i k x}}{E-\tilde{H}(k)}
$$

and

$$
\frac{1}{\alpha}=G(0)=\int \frac{d^{2} \vec{k}}{(2 \pi)^{2}} \frac{1}{E-\tilde{H}_{2 d}(\vec{k})},
$$

where $\tilde{H}_{2 d}(\vec{k})$ is the $2 \mathrm{D}$ Hamiltonian in the momentum representation. For this section, we assume an isotropic Hamiltonian $\tilde{H}_{2 d}(\vec{k})=\tilde{H}_{2 d}(k)$. Hence we can write Eq. (9) as

$$
\frac{1}{\alpha}=G(0)=\int \frac{d \theta}{2 \pi} \int_{0}^{\infty} \frac{k d k}{2 \pi} \frac{1}{E-\tilde{H}_{2 d}(k)} .
$$

Equation (10) cannot be simply interpreted in terms of plane wave matching like its $1 \mathrm{D}$ counterpart since contour integration over a complex $k$ cannot be used: the integration range is $0 \leqslant k<\infty$. Nevertheless, we can proceed using the Kramers-Kronig relation along with the symmetry of the imaginary part of the integrand and obtain an expression analogous to Eq. (4).

The Kramers-Kronig relation connects the imaginary and real parts of a complex function $F(s)$ which is analytical in the upper half plane, $\operatorname{Im}(s)>0$, and falls off faster than $1 /|s|$. To apply it here, we define

$$
F(s)=\int_{0}^{\infty} \frac{k d k}{2 \pi} \frac{e^{i k s}}{E-\tilde{H}_{2 d}(k)} .
$$

Note that this function is not the Green's function in real space since there is no angular dependence taken into account in the exponent. However, $F(0)=G(0)$. Furthermore, we see that only $\operatorname{Re}[G(0)]$ plays a role in determining the boundstate energy of an impurity state. We use the Kramers-Kronig relation to write

$$
\operatorname{Re}[F(0)]=\int_{-\infty}^{\infty} \frac{d s^{\prime}}{\pi s^{\prime}} \operatorname{Im}\left[F\left(s^{\prime}\right)\right] .
$$

This is helpful since the imaginary part of $F(s)$ for $\operatorname{Im}(\mathrm{s})=0$ obeys

$$
\operatorname{Im}[F(s)]=\frac{1}{2} \int_{-\infty}^{\infty} \frac{k d k}{2 \pi} \frac{e^{i k s}}{E-\tilde{H}_{2 d}(k)},
$$


with the $k$ integral now stretching over the entire real axis. It is assumed that the Hamiltonian $\tilde{H}_{2 d}(k)$ is an even function of $\mathrm{k}$.

After these steps we can rewrite the bound-state energy condition as

$$
\begin{aligned}
\frac{1}{\alpha} & =\frac{1}{\pi} \int_{-\infty}^{\infty} \frac{d s}{s} \operatorname{Im}\left[\int_{0}^{\infty} \frac{k d k}{2 \pi} \frac{e^{i k s}}{E-\tilde{H}_{2 d}(k)}\right] \\
& =\frac{1}{\pi} \int_{0}^{\infty} \frac{d s}{i s}\left[\int_{-\infty}^{\infty} \frac{k d k}{2 \pi} \frac{e^{i k s}}{E-\tilde{H}_{2 d}(k)}\right] .
\end{aligned}
$$

Finally, we also eliminate the factor of $k$ in the integrand using a derivative with respect to $s$ to yield our final expression:

$$
\frac{1}{\alpha}=\frac{-1}{\pi} \int_{0}^{\infty} \frac{d s}{s} \frac{\partial}{\partial s}\left[\int_{-\infty}^{\infty} \frac{d k}{2 \pi} \frac{e^{i k s}}{E-\tilde{H}_{2 d}(k)}\right] .
$$

The term in the square brackets in Eq. (16) is completely analogous to the expression used to find the bound-state energy of a 1D solution of a point impurity [Eq. (2)] and we therefore define

$$
G_{1 d}(s)=\int_{-\infty}^{\infty} \frac{d k}{2 \pi} \frac{e^{i k s}}{E-\tilde{H}_{2 d}(k)},
$$

which can be viewed as the Green's function of a 1D Hamiltonian $H_{1 d}(s) \equiv \tilde{H}_{2 d}\left(k \rightarrow \frac{\partial}{i \partial s}\right)$ in real space. Qualitatively, the effective real-space 1D Hamiltonian $H_{1 d}$ describes a single direction of the 2D momentum-space Hamiltonian $\tilde{H}_{2 d}$.

The final result of this reasoning is that the bound-state energy $E$ for a 2D point impurity can be obtained by solving a 1D impurity problem with a modified potential. Provided that $G_{1 d}(x)$ is the solution of the 1D Hamiltonian $H_{1 d}(x)$, the wave function $\psi_{1 d}(x)=G_{1 d}(x)$ up to an overall normalization factor for a scalar Hamiltonian. From Eqs. (16) and (17) the effective 1D Schrödinger equation can be written with a modified impurity potential:

$$
\left[E-H_{1 d}(x)\right] \psi_{1 d}(x)=\delta(x) \alpha^{\prime} \psi_{1 d}(0),
$$

with $\alpha^{\prime}$ given by

$$
\alpha^{\prime}=-\alpha\left[\frac{1}{\pi} \int_{0}^{\infty} \frac{d s}{s} \frac{\partial \psi_{1 d}(s)}{\partial s}\right] \frac{1}{\psi_{1 d}(0)} .
$$

$\psi_{1 d}(s)$ on the right-hand side of Eq. (19) emerges as the solution of the effective 1D problem but does not appear to have any physical significance.

\section{Example: Free particle}

The first example for our method is the free particle with quadratic dispersion. We demonstrate the method by finding the $1 \mathrm{D}$ effective Hamiltonian in real space and solving it in the spirit of the wave-function-matching method. The free-particle example in two dimensions is a bit pathological, however, since a high-energy cutoff is required and the bound-state energies of point impurities depend on it. This is seen by inspecting Eq. (9): the momentum integration diverges unless a cutoff is imposed. This introduces a technical challenge for our quest to use momentum integration over the entire real axis. We resolve it by introducing the cutoff using the Pauli-Villars regularization technique [22].
Starting with the free Hamiltonian and an impurity potential,

$$
\begin{gathered}
H_{2 d}=-\frac{\nabla^{2}}{2 m}, \\
V_{\text {imp }}=\alpha \delta^{(2)}(\vec{x}),
\end{gathered}
$$

we write the Green's-function condition for an impurity bound state and include the Pauli-Villars regulators $W^{2} /\left(k^{2}+W^{2}\right)$ :

$$
\frac{1}{\alpha}=\int \frac{d^{2} \vec{k}}{(2 \pi)^{2}} \frac{1}{E-\tilde{H}_{2 d}(k)}\left(\frac{W^{2}}{W^{2}+k^{2}}\right) .
$$

According to our recipe, this is equivalent to finding bound states of the following 1D effective Hamiltonian:

$$
\tilde{H}_{1 d}(k)=\frac{k^{2}}{2 m}-\left(E-\frac{k^{2}}{2 m}\right) \frac{k^{2}}{W^{2}} .
$$

It is interesting to consider the effective 1D Hamiltonian, Eq. (23), and the effect that the Pauli-Villars regulator has. $\tilde{H}_{1 d}$ preserves the original dispersion relation, and a $k^{2} / 2 m$ pole is reflected in the Green's function. In addition, the Green's function acquires an additional pair of poles at $k_{p}= \pm i \mathrm{~W}$.

Now we can treat the problem just like a $1 \mathrm{D}$ problem with a plane-wave solution but with multiple plane waves corresponding to all solutions of $E \psi_{1 d}=\tilde{H}_{1 d} \psi_{1 d}$ (which correspond to the poles of the Green's function). The solution must satisfy continuity conditions at $x=0$ of the derivatives:

$$
\left[\frac{\partial^{m} \psi(x)}{\partial x^{m}}\right]_{0-}^{0+}=0
$$

for $m=0,1,2$, which provides enough conditions to fix the weights of the plane waves up to an overall factor for $x<0$ and $x>0$ :

$$
\begin{gathered}
\psi(x>0)=\frac{1}{\lambda_{1}} e^{-\lambda_{1} x}-\frac{1}{\lambda_{2}} e^{-\lambda_{2} x}, \\
\psi(x<0)=\frac{1}{\lambda_{1}} e^{\lambda_{1} x}-\frac{1}{\lambda_{2}} e^{\lambda_{2} x} .
\end{gathered}
$$

The exponents are $\lambda_{1}=\sqrt{-2 m E}$ and $\lambda_{2}=W$, which are the poles of Green's function. Now we use Eq. (18) to find the bound-state energy for a given impurity potential strength. First, we carry out the integration that yields the effective $\alpha^{\prime}$ using Eq. (19):

$$
\alpha^{\prime}=\frac{\alpha}{\pi} \frac{\lambda_{1} \lambda_{2}}{\lambda_{2}-\lambda_{1}} \log \left(\frac{\lambda_{2}}{\lambda_{1}}\right) .
$$

With this modified impurity potential and the $1 \mathrm{D}$ effective Hamiltonian equation (23) in real space, one can solve the 1D problem and get

$$
\frac{1}{\alpha}=\frac{-m / \pi}{1+2 m E / W^{2}} \log \left(\frac{W}{\sqrt{-2 m E}}\right) .
$$

As seen from Eq. (28), the bound-state energy is renormalized by the cutoff and mass, but its qualitative behavior as a function of the impurity potential strength is unchanged. This is depicted in Fig. 2. One may easily check that Eq. (28) obtained in our method exactly agrees with the corresponding equation obtained with the direct integration over momenta in Eq. (22). 


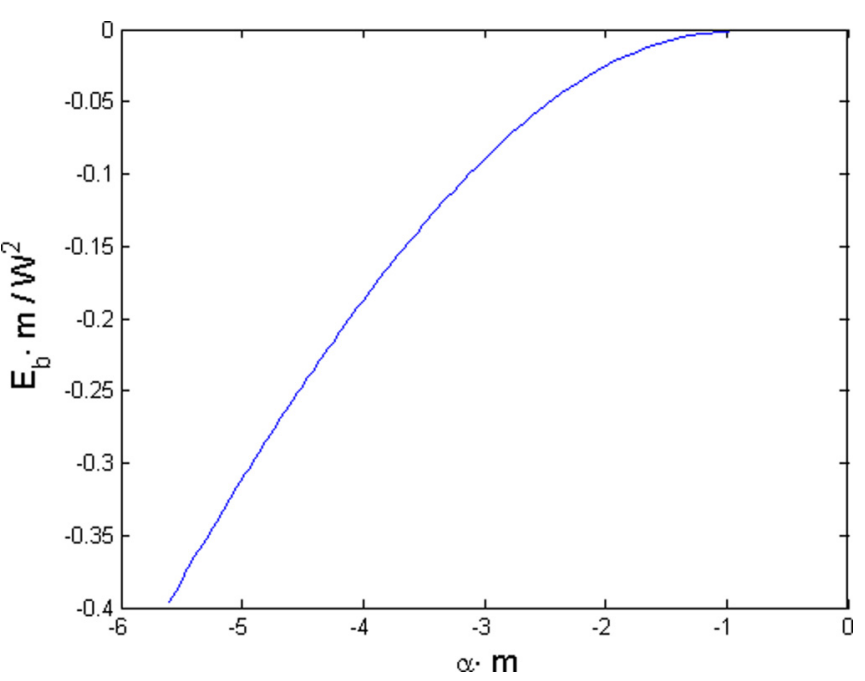

FIG. 2. (Color online) The bound energy $E_{b}$ of the free-particle model with mass $m$ and cutoff $W$ for the regularization is plotted as a function of impurity strength $\alpha$. The bound energy decreases as the impurity strength negatively increases. In the limit of zero impurity strength, the bound energy converges to zero.

\section{A particle in a Mexican-hat-shaped band}

Let us study one more isotropic Hamiltonian, a Mexicanhat-shaped energy band:

$$
\tilde{H}_{2 d}(k)=J\left(k^{2}-k_{0}^{2}\right)^{2},
$$

where $J$ is a constant with units of length cubed. The band covers all positive energies and therefore the energy of any bound state should be negative.

The Mexican-hat Hamiltonian does not have a UV divergence problem like the free-particle Hamiltonian and therefore does not require a Pauli-Villars regulator. Thus $\tilde{H}_{1 d}(k)=$ $\tilde{H}_{2 d}(k)$ in this case. Incidentally, it also has the same order of derivatives as the regularized free-particle Hamiltonian, Eq. (23). Therefore, it obeys the same number of continuity relations, and we may simply use the same functional form ansatz as in Eqs. (25) and (26), with the wave numbers given by

$$
\begin{aligned}
& k_{1}=i \lambda_{1}=k_{0}\left(1+i \sqrt{\frac{-E}{J k_{0}^{4}}}\right)^{1 / 2}, \\
& k_{2}=i \lambda_{2}=k_{0}\left(1-i \sqrt{\frac{-E}{J k_{0}^{4}}}\right)^{1 / 2},
\end{aligned}
$$

which are the result of setting $E=\tilde{H}_{1 d}(k)$. Carrying out the same steps as in the free-particle case, we obtain the relation between the bound-state energy and the impurity strength:

$$
\begin{aligned}
\frac{1}{\alpha} & =\frac{1}{2 \pi J} \frac{\log \left(\lambda_{2} / \lambda_{1}\right)}{\lambda_{2}^{2}-\lambda_{1}^{2}} \\
& =\frac{-1}{4 \pi J} \frac{\tan ^{-1} \sqrt{-E / J k_{0}^{4}}}{\sqrt{-E / J}},
\end{aligned}
$$

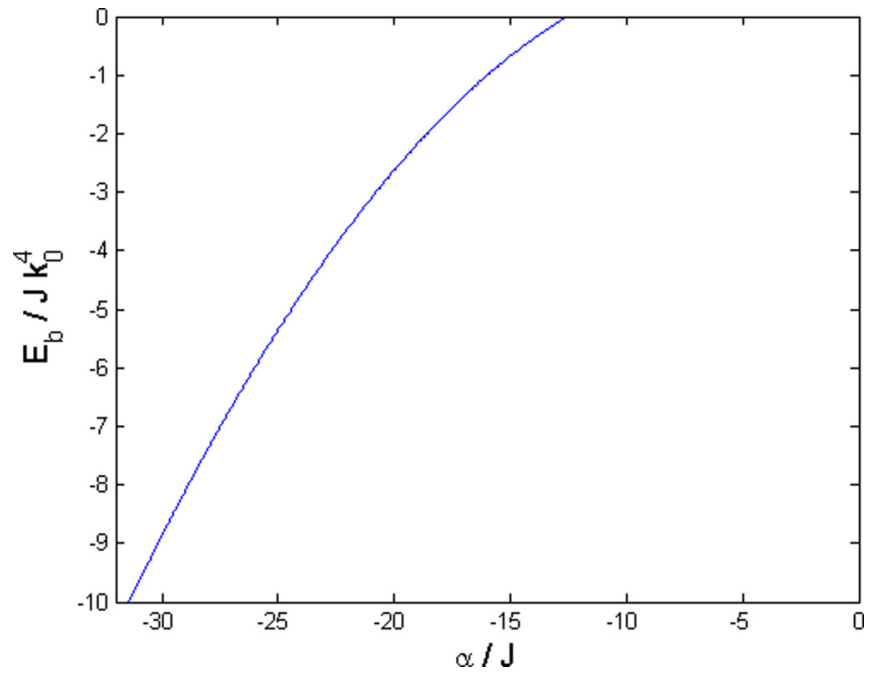

FIG. 3. (Color online) The bound energy $E_{b}$ of the Mexican-hat model is plotted as a function of impurity strength $\alpha$. The general behavior is similar to that of the free-particle model except that the bound state appears when $\alpha<-1 / 4 \pi J k_{0}^{2}$.

where it follows from the above expression that the bound state exists only for an attractive potential. The result is plotted in Fig. 3 with renormalized axes.

\section{WAVE-FUNCTION MATCHING FOR ANISOTROPIC BANDS}

When the impurity problem is anisotropic, we can no longer solve for the bound-state energies exactly using only a small number of incoming and outgoing beams. Instead, we can still consider simple 1D wave functions for the radial part of the problem and then consider a superposition over all angles. Here we extend the wave-function-matching method to such anisotropic systems.

We begin by writing the Hamiltonian in polar momentum coordinates: $\tilde{H}_{2 d}(\vec{k})=\tilde{H}_{2 d}^{\theta}(k)$, with $\theta$ being the momentum direction. Now, when going from Eq. (9) to Eq. (10), the angular integration must be kept:

$$
\frac{1}{\alpha}=\int \frac{d \theta}{2 \pi} \int_{0}^{\infty} \frac{k d k}{2 \pi} \frac{1}{E-\tilde{H}_{2 d}^{\theta}(k)} .
$$

Following exactly the same steps, we introduce a 1D wave function along momentum angle $\theta$ :

$$
G_{1 d}^{\theta}(s)=\int_{-\infty}^{\infty} \frac{d k}{2 \pi} \frac{e^{i k s}}{E-\tilde{H}_{2 d}^{\theta}(k)},
$$

which is the Green's function of the 1D Hamiltonian $H_{1 d}^{\theta}(s) \equiv \tilde{H}_{2 d}^{\theta}\left(k \rightarrow \frac{\partial}{i \partial s}\right)$. The modified relation between potential strength and associated bound-state energy is

$$
\frac{1}{\alpha}=\int \frac{d \theta}{2 \pi} \frac{1}{\alpha^{\theta}},
$$

where $1 / \alpha^{\theta}$, just like on the right-hand side of Eq. (16), is

$$
\frac{1}{\alpha^{\theta}}=-\frac{1}{\pi} \int_{0}^{\infty} \frac{d s}{s} \frac{\partial G_{1 d}^{\theta}(s)}{\partial s} .
$$


As pointed out above, the Green's function $G_{1 d}^{\theta}(s)$ is constructed using plane-wave solutions of $\tilde{H}_{2 d}^{\theta}\left(\frac{\partial}{i \partial s}\right)$, treated as a 1D Hamiltonian. For a general anisotropic system, the above prescription requires infinitely many directions of $1 \mathrm{D}$ solutions.

A more direct formulation of the $\alpha_{\theta}$ in terms of a 1D Schrödinger equation for each $\theta$ direction is as follows. We construct for each $\theta$ a solution of the 1D bound-state equation,

$$
\left[E-\tilde{H}_{\theta}\left(\frac{\partial}{i \partial s}\right)\right] \psi^{\theta}(s)=\alpha^{\prime \theta} \delta(s) \psi^{\theta}(s),
$$

and find $\alpha^{\prime \theta}$ in terms of $E$ by requiring that the 1D problem has a bound state at energy $E$. The integrand on the right-hand side of Eq. (36), $\alpha^{\theta}$, is given in terms of $\alpha^{\prime \theta}$ and the impurity-state wave function $\psi^{\theta}(s)$ as

$$
\frac{1}{\alpha^{\theta}}=-\frac{1}{\alpha^{\prime \theta}} \frac{1}{\pi} \int_{0}^{\infty} \frac{d s}{s} \frac{\partial \psi^{\theta}(s)}{\partial s} \frac{1}{\psi^{\theta}(0)} .
$$

\section{A. Anisotropic mass}

As a simple example, we consider impurity states in a band described by a parabolic dispersion with an anisotropic mass:

$$
\tilde{H}(\vec{k})=\frac{k_{x}^{2}}{2 m_{x}}+\frac{k_{y}^{2}}{2 m_{y}} \equiv \frac{k^{2}}{2 m(\theta)},
$$

where

$$
\frac{1}{m(\theta)}=\frac{\cos ^{2} \theta}{m_{x}}+\frac{\sin ^{2} \theta}{m_{y}} .
$$

This problem can be simply solved by rescaling $x$ relative to $y$ and obtaining the isotropic solution. Therefore this calculation should just be a demonstration of using our method. For more complicated band structures this will not be possible (e.g., bands with multiple minima, as discussed below).

Let us now construct $G_{1 d}^{\theta}$ and $\alpha^{\theta}$. For a given direction we can make use of the relation we obtained before for a free particle, using $m(\theta)$ instead of $m$. Using Eq. (28), with $\alpha \rightarrow \alpha^{\theta}$, we obtain

$$
\frac{1}{\alpha^{\theta}}=\frac{-m(\theta) / \pi}{1+2 m(\theta) E / W^{2}} \log \left(\frac{W}{\sqrt{-2 m(\theta) E}}\right) .
$$

The last step in this consideration should be the integration of Eq. (42) over all momentum directions. However, we find that in this case it is enough to consider a pair of perpendicular directions, the extrema of $\alpha^{\theta}$ which occur at $\theta=0, \pi$ and $\theta=$ $\pi / 2,3 \pi / 2$. This is shown in Fig. 4 where the dash-dotted black line shows the results of the integration over all directions and the other lines show two different directions (solid and dotted lines) and their combination (dashed line). As evident from the graph, the combination of two perpendicular directions is very similar to the integrated expression. This is equivalent to evaluating the $\theta$ integral using a discrete sum, which could be used to reduce the number of beams necessary in a $2 \mathrm{D}$ problem to a finite and small number.

\section{A BAND WITH MULTIPLE MINIMA}

Perhaps the most interesting application of our method is to dispersion relations which contain several gap minima. The

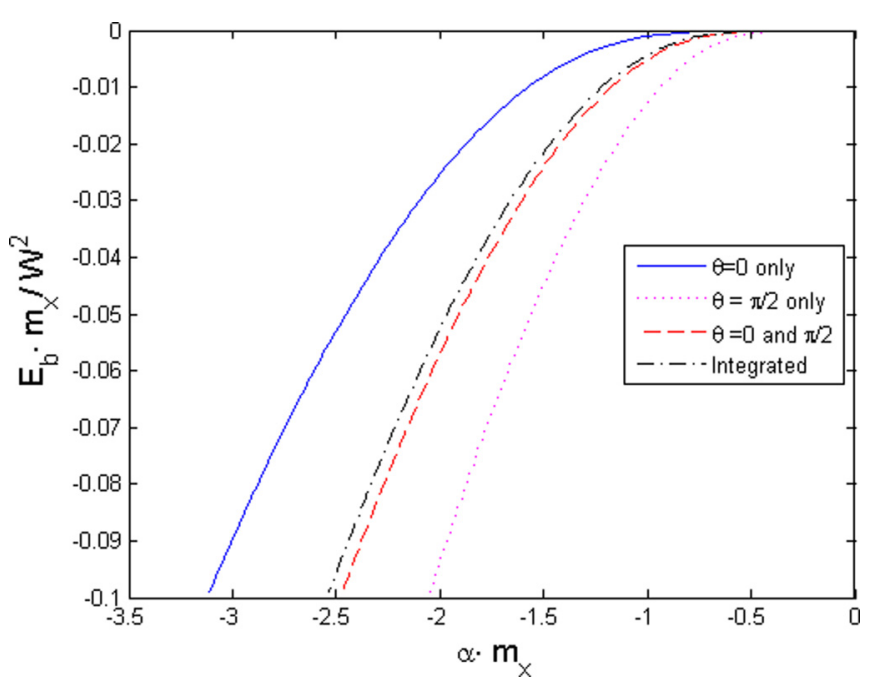

FIG. 4. (Color online) Bound energy associated with a single impurity for a two-mass anisotropic band model $\left(m_{y} / m_{x}=2\right)$. The exact bound-state energy is obtained by integrating over all momentum angles $\theta$, while taking a straight " $1 \mathrm{D}$ " path with a certain momentum angle yields slightly deviated bound-state energy. Nevertheless, the consideration of only two momentum angles $\theta=0$ and $\pi / 2$ gives a fair agreement with the exact result.

qualitative picture of a bound state consisting of a simple superposition of several 1D beams each coming from the vicinity of one particular gap minimum is rather intuitive and appealing. Our method allows making this picture quantitative even for complicated band structures.

So far we have identified the single minimum of the band and used an approximated version of the Hamiltonian around the minimum. Then, according to Eq. (9), the Green's function is obtained and integrated with an appropriate cutoff $W$ introduced through the Pauli-Villars regulator. The generalization to more than one band minimum is done by dividing the integration in Eq. (9) into the summation of multiple integrations with different local Hamiltonians, which are the expansions of the Hamiltonian around each minimum. In many cases where multiple valleys need to be considered, the wave function is a multidimensional spinor. Therefore, we need to represent the impurity strength $\alpha$ by a matrix and use the determinant condition for bound states, Eq. (5).

$$
\int \frac{d^{2} \vec{k}}{(2 \pi)^{2}} \frac{1}{E-\tilde{H}_{2 d}(\vec{k})} \approx \sum_{l} \int \frac{d^{2} \vec{k}_{l}}{(2 \pi)^{2}} \frac{1}{E-\tilde{H}_{l}\left(\vec{k}_{l}\right)}
$$

where $l$ goes over the band minima and $H_{l}$ is the expansion of the Hamiltonian around the $l$ th minimum with the appropriate Pauli-Villars regularization included. The origin of each $\vec{k}_{l}$ is set to the center of the minima such that the "valley" Hamiltonian $\tilde{H}_{l}\left(\vec{k}_{l}\right)$ has maximum symmetry. Thus, the condition for a bound state is

$$
\operatorname{det}\left(1-\sum_{l} \int \frac{d^{2} \vec{k}_{l}}{(2 \pi)^{2}} \frac{1}{E-\tilde{H}_{l}\left(\vec{k}_{l}\right)} \alpha\right)=0 .
$$

Applying the Kramers-Kronig trick here as well, like in the anisotropic construction, we write the potential strength as a 
sum:

$$
\operatorname{det}\left(1-\sum_{l} \int \frac{d \theta}{2 \pi} \frac{1}{\alpha_{l}^{\theta}} \alpha\right)=0
$$

where $\left(\alpha_{l}^{\theta}\right)^{-1}$ is a matrix, given by

$$
\frac{1}{\alpha_{l}^{\theta}}=-\frac{1}{\pi} \int_{0}^{\infty} \frac{d s}{s} \frac{\partial G_{l}^{\theta}(s)}{\partial s},
$$

with

$$
G_{l}^{\theta}(s)=\int_{-\infty}^{\infty} \frac{d k}{2 \pi} \frac{e^{i k s}}{E-\tilde{H}_{l}^{\theta}(k)},
$$

which is the Green's function for the Hamiltonian $\tilde{H}_{l}^{\theta}=$ $\tilde{H}_{l}(k \hat{x} \cos \theta+k \hat{y} \sin \theta)$.

As described in Sec. III, Eq. (46) can also be interpreted in terms of individual 1D plane waves in each direction and valley. This is made a bit more complicated by taking into account a spinor index. Denoting the spinor indices by $\sigma, \sigma^{\prime}$, we have

$$
\left(\alpha_{l}^{\theta}\right)_{\sigma \sigma^{\prime}}^{-1}=-\frac{1}{b_{l}^{\theta, \sigma^{\prime}}} \frac{1}{\pi} \int_{0}^{\infty} \frac{d s}{s} \frac{\partial\left[\psi_{l}^{\theta, \sigma^{\prime}}\right]_{\sigma}(s)}{\partial s} \frac{1}{\left[\psi_{l}^{\theta, \sigma^{\prime}}\right]_{\sigma^{\prime}}(0)},
$$

where $b_{l}^{\theta, \sigma^{\prime}}$ and $\left[\psi_{l}^{\theta, \sigma^{\prime}}\right]_{\sigma}(s)$ are obtained by solving the 1D impurity problem of a particle with the Hamiltonian $\tilde{H}_{l}^{\theta}(k \rightarrow$ $\left.\frac{\partial}{i \partial s}\right)$, which is the original 2D Hamiltonian for a particular momentum direction $\theta$ and with momentum in the vicinity of the bottom of valley $l$. Let us clarify these symbols further and state the impurity problem that needs to be solved:

$$
\left[\left(E-\tilde{H}_{l}^{\theta}\right) \psi_{l}^{\theta, \sigma^{\prime}}\right]_{\sigma}(s)=\delta_{\sigma \sigma^{\prime}} \delta(s) b_{l}^{\theta, \sigma^{\prime}}\left[\psi_{l}^{\theta, \sigma^{\prime}}\right]_{\sigma}(s) .
$$

The index $\sigma^{\prime}$ indicates to which component the impurity couples, and $b_{l}^{\theta, \sigma^{\prime}}$ is the impurity strength required to induce an impurity state with energy $E$ in the specified valley, direction, and component of the spinor involved. Accordingly, $\left[\psi_{l}^{\theta, \sigma^{\prime}}\right]_{\sigma}(s)$ is the $\sigma$ component at point $s$ of the (valley $l$ and momentum angle $\theta$ ) wave function of a bound state of an impurity that couples to the $\sigma^{\prime}$ component. Note that the impurity strength is multiplied by the $\sigma^{\prime}$ component of the wave function.

\section{A. Kane-Mele model}

As an example of an anisotropic system with multiple minima we consider an impurity problem in the Kane-Mele model [23]. This model describes electrons hopping on a honeycomb lattice with mirror-symmetric spin-orbit coupling. It was the first model theorized to display a time-reversalsymmetric topological phase, i.e., the quantum spin Hall phase. The two sublattices of the honeycomb lattice are encoded in two-dimensional spinors, and its band structure has two massive Dirac points in the Brillouin zone. For simplicity, but without loss of generality, we focus on a nonmagnetic impurity and consider spinless fermions. The Hamiltonian [24] is

$$
H=t \sum_{\langle i j\rangle} c_{i}^{\dagger} c_{j}+i \lambda_{S O} \sum_{\langle\langle i j\rangle\rangle} v_{i j} c_{i}^{\dagger} s^{z} c_{j}
$$

The first term is a nearest-neighbor hopping, and the second term is spin-orbit interaction. $s^{z}$ is a Pauli matrix which acts in the spin space. $v_{i j}=(2 / \sqrt{3})\left(\hat{d}_{1} \times \hat{d}_{2}\right)_{z}= \pm 1$, where $\hat{d}_{1}$ and $\hat{d}_{2}$ are unit vectors along the two bonds that the electron traverses going from site $j$ to its next nearest neighbor $i$. The effective Hamiltonian near the valley $K$ can be expressed by a $2 \times 2$ matrix in the pseudospin basis:

$$
\tilde{H}_{K}(q)=\left(\begin{array}{cc}
m & q e^{-i \theta} \\
q e^{i \theta} & -m
\end{array}\right),
$$

where $q$ is the momentum measured from $K$. Since the linear spectrum is not well behaved in the presence of a $\delta$ function potential, we employ the Pauli-Villars regularization procedure as before. The regularized Hamiltonian reads

$$
\tilde{H}_{K}^{\prime}(q)=\tilde{H}_{K}(q)-\left[E-\tilde{H}_{K}(q)\right] \frac{q^{2}}{W^{2}} .
$$

The impurity is also described by a $2 \times 2$ potential matrix, and we choose to put it on the $A$ site.

$$
\alpha=\alpha_{0}\left(\begin{array}{ll}
1 & 0 \\
0 & 0
\end{array}\right) .
$$

To work this Hamiltonian in the sprit of the $1 \mathrm{D}$ wavefunction-matching method, we ought to solve the real-space 1D Hamiltonian $H_{K}^{\prime}(s)=\tilde{H}_{K}^{\prime}\left(q \rightarrow \frac{\partial}{i \partial s}\right)$. Instead, let us work with the real-space Green's function and then make use of Eq. (5) to find an associate bound energy of a single impurity. We follow the matrix version of Eq. (35):

$$
\begin{aligned}
G_{K}^{\theta}(s) & =\int \frac{d k}{2 \pi} \frac{e^{i k s}}{E-\tilde{H}_{K}^{\prime}(k)} \\
& =\sum_{j=1,2} \frac{(-1)^{j} e^{-\lambda_{j} s} / 2}{\left|\lambda_{j}\right|\left(1+\frac{E^{2}-m^{2}}{W^{2}}\right)}\left(\begin{array}{cc}
E+m & i \lambda_{j} e^{-i \theta} \\
i \lambda_{j} e^{i \theta} & E-m
\end{array}\right),
\end{aligned}
$$

where the poles of the Green's function are $\lambda_{1}=\sqrt{m^{2}-E^{2}}$ and $\lambda_{2}=W$ for $s>0$ and it is understood that $(-1)^{j=1}=-1$ and $(-1)^{j=2}=1$. The diagonal elements of the solution are symmetric around $s$, while the off-diagonal elements are asymmetric. This is the wave function for the two-sublattice system. For the other Dirac valley $K^{\prime}$, the calculation is similar, except the sign of the spin-orbit coupling is opposite. Following Eq. (37) for anisotropic Hamiltonians,

$$
\frac{1}{\alpha_{K, K^{\prime}}^{\theta}}=\frac{-1}{\pi} \int_{0}^{\infty} \frac{d s}{s} \frac{\partial G_{K, K^{\prime}}^{\theta}(s)}{\partial s} .
$$

The only remaining step is to consider the determinant from Eqs. (5) and (44) in order to connect the potential strength and bound-state energy:

$$
\operatorname{Det}\left[I_{2}+\sum_{m=K, K^{\prime}} \int \frac{d \theta}{2 \pi} \frac{1}{\alpha_{m}^{\theta}} \alpha\right]=0 .
$$

As a result, we obtain the relation between the bound-state energy and the potential strength $\alpha_{0}$ in the Kane-Mele model:

$$
\frac{1}{\alpha_{0}}=\frac{E}{2 \pi^{2}\left(W^{2}-m^{2}+E^{2}\right)} \log \left(\frac{W}{\sqrt{m^{2}-E^{2}}}\right) .
$$



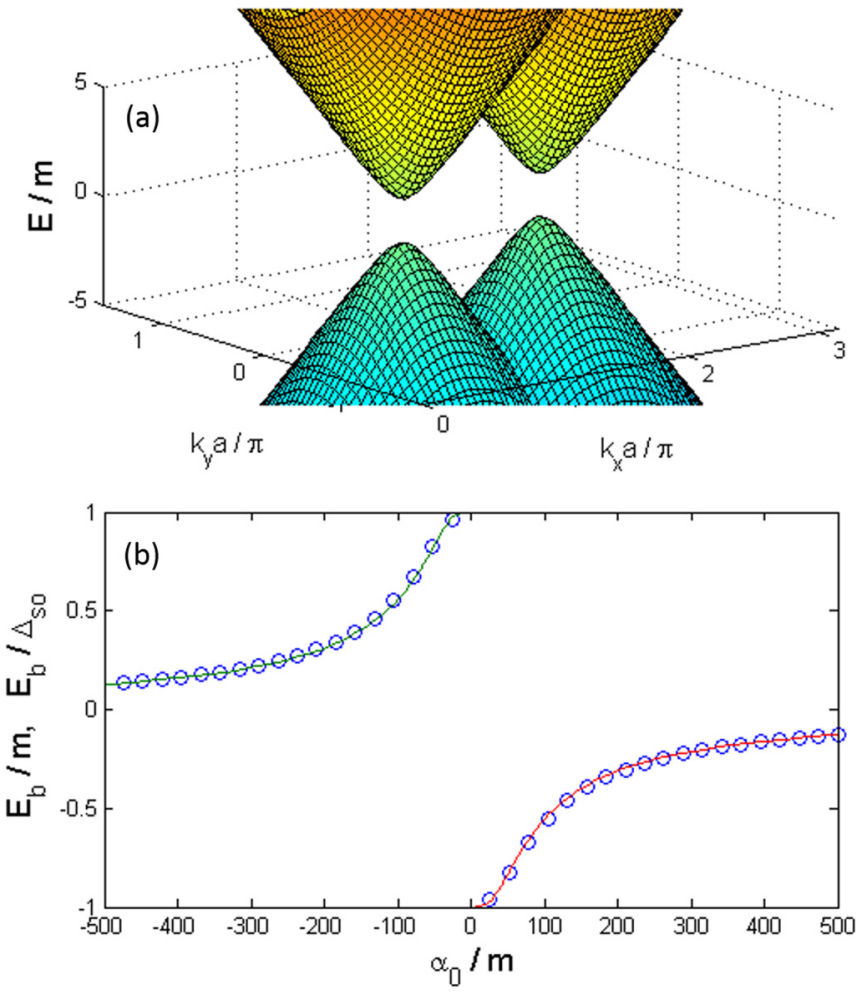

FIG. 5. (Color online) (a) The Kane and Mele model is employed as an example of a multiple-minima band with $W / m=1.6$. The band gap appears near zero energy, and its size is proportional to the spin-orbit coupling. The bound-state energy associated with a single impurity is always within the gap, and the energies converge to zero energy at infinite impurity strength. (b) The exact diagonalization result of the Kane-Mele model on a honeycomb lattice of $20 \times 20$ unit cells is overlaid for comparison with $\lambda_{S O}=m / 6 \sqrt{3}$ (open circles) so that the band gap of the lattice model $\Delta_{S O}=6 \sqrt{3} \lambda_{S O}=m$. The cutoff $W$ is chosen such that the dispersion relation of the continuum model is a good approximation of that of the lattice model.

This is plotted in Fig. 5, where we show both positive and negative impurity strengths and the associated positive and negative bound-state energies. We compare our results for an exact diagonalization solution for the bound-state energy.

\section{GENERALIZATION TO HIGHER DIMENSIONS}

We have studied how to obtain the bound energy associated with a single impurity and found that the Green's function at the impurity potential is a crucial quantity to find:

$$
G(\overrightarrow{0})=\int \frac{d \Omega}{(2 \pi)^{d-1}} \int_{0}^{\infty} \frac{k^{d-1} d k}{2 \pi} \frac{1}{E-\tilde{H}_{d}(\vec{k})} .
$$

Although we have only discussed 2D systems, our method has a straightforward extension to higher dimensions. For odd-dimensional cases, the wave function can be expressed by the summation of plane waves with certain momentum angles since the $k$ integrals in Eq. (58) could be extended to the entire real $k$ range. Then poles of the effective oriented Green's function combine to produce the solution, and the wave-function-matching picture straightforwardly follows when relating it to the impurity strength.

In this section let us consider a Hamiltonian in an evendimensional space. For simplicity we assume an isotropic Hamiltonian without any spinor structure. Then the boundstate energy associated with the single-impurity potential, $V(\vec{x})=\alpha \delta^{2 n}(\vec{x})$, is expressed similar to Eq. (9) by

$$
\begin{aligned}
\frac{1}{\alpha} & =\int \frac{d^{2 n} \vec{k}}{(2 \pi)^{2 n}} \frac{1}{E-\tilde{H}_{2 n}(k)} \\
& =\int \frac{k^{2 n-1} d k}{2 \pi} \frac{1}{E-\tilde{H}_{2 n}(k)},
\end{aligned}
$$

where the difficulty lies in the integration in polar coordinates; as in the $2 \mathrm{D}$ case, the contour integration is not readily possible, and therefore any connection to a semiclassical beam analysis is not possible. Nevertheless, to overcome this hurdle we may use the same prescription as in two dimensions. We use the Kramers-Kronig relation to make use of the symmetric imaginary part of the integrand and obtain the generalized relation:

$$
\frac{1}{\alpha}=\frac{-1}{\pi} \int_{0}^{\infty} \frac{d s}{s} \frac{\partial^{2 n-1}}{\partial s^{2 n-1}}\left[\int_{-\infty}^{\infty} \frac{d k}{2 \pi} \frac{e^{i k s}}{E-\tilde{H}_{2 n}(k)}\right] .
$$

For isotropic and single-minimum-band-type problems, the above relation can be translated to finding an impurity state in a renormalized-strength impurity potential in the 1D effective Schrödinger equation:

$$
\left[E-H_{1 d}\left(\partial_{x}\right)\right] \psi_{1 d}(x)=\delta(x)\left[\frac{-\alpha}{\pi} \int_{0}^{\infty} \frac{d s}{s} \frac{\partial^{2 n-1}}{\partial s^{2 n-1}} \psi_{1 d}(s)\right]
$$

where $H_{1 d}\left(\partial_{x}\right)=\tilde{H}_{2 n}\left(k \rightarrow \frac{\partial}{i \partial x}\right)$ and the solution is the sum of plane waves with complex wave numbers:

$$
\psi_{1 d}(x)=\int_{-\infty}^{\infty} \frac{d k}{2 \pi} \frac{e^{i k s}}{E-\tilde{H}_{2 n}(k)} .
$$

\section{CONCLUSIONS}

Finding impurity bound states in one dimension is quite intuitive and is carried out by combining plane-wave states into a consistent solution. The plane-wave approach to solving impurity problems in one dimension arises naturally when considering the $T$-matrix approach or, equivalently, the Green's function expression of Eq. (4). The contour integration of the 1D Green's function results in a discrete sum of pole contributions, each of which is associated with the plane-wave eigenstate of the uniform Hamiltonian. In one dimension, the Schrödinger equation can be used directly to obtain impurity states, and such a calculation would involve satisfying matching conditions at the impurity location of plane-wave solutions (the same that arise from the poles of the Green's function) belonging to either side. The intuitive interpretation of impurity states as a simple combination of plane waves is completely lost at higher (even) dimensions, which is seen technically by not being able to reduce Eq. (4) to the sum of residues of the Green's function.

In this paper we presented an approach for finding bound states which reduces the impurity problem in any dimension 
to $1 \mathrm{D}$ impurity problems and thus allows an interpretation in terms of a small set of incoming and outgoing plane waves on a linear trajectory.

We demonstrated that the method could be efficiently used to find impurity bound states in a general band structure where the gap could have multiple minima and a spinor structure. Our method relied on the use of the Kramers-Kronig relation, which maps the Green's-function formula at any dimension to an expression which is given again by a sum of residues corresponding to plane-wave solutions of the pure model.

Presenting a few examples, we demonstrated how our method easily lends itself to approximating lattice Hamiltonians in terms of a discrete sum of separate valley Hamiltonians. As we show, when Pauli-Villars regulators are used to provide a cutoff for valley Hamiltonians, we can still use the Kramers-Kronig relation to connect bound states with a discrete sum of plane waves. The Pauli-Villars regulators, however, add additional poles to the Green's function of the pure system, which also need to be included in the plane-wave superposition.
While we only demonstrated the method in two dimensions, the wave-function-matching method can be extended to any dimension. All odd-dimensional systems are analogous to the $1 \mathrm{D}$ case with additional angular variables, and all evendimensional systems are analogous to the $2 \mathrm{D}$ case. This is because the wave-function-matching method is closely connected to the contour integration, which reduces the problem to finding poles of the Green's function; the contour integration applies in odd dimensions but not in even ones. For most systems without rotational symmetry of local Hamiltonians, only a few momentum angles are necessary to complete the semiclassical interpretation of a bound state.

\section{ACKNOWLEDGMENTS}

K.W.K. and G.R. would like to acknowledge support from DARPA through FENA, as well as from the IQIM, an NSF center with the support of the Gordon and Betty Moore Foundation. T.P.B. would like to acknowledge support from NSERC and FQRNT.
[1] P. W. Anderson, Phys. Rev. 109, 1492 (1958).

[2] P. W. Anderson, Rev. Mod. Phys. 50, 191 (1978).

[3] E. Abrahams, 50 Years of Anderson Localization (World Scientific, Singapore, 2010).

[4] F. Evers and A. D. Mirlin, Rev. Mod. Phys. 80, 1355 (2008).

[5] P. Anderson, J. Phys. Chem. Solids 11, 26 (1959).

[6] B. T. Matthias, H. Suhl, and E. Corenzwit, Phys. Rev. Lett. 1, 152 (1958).

[7] A. A. Abrikosov and L. P. Gor'kov, Sov. Phys. JETP 8, 1090 (1959).

[8] D. Markowitz and L. P. Kadanoff, Phys. Rev. 131, 563 (1963).

[9] A. Larkin, JETP Lett. 2, 130 (1965).

[10] M. Ma and P. A. Lee, Phys. Rev. B 32, 5658 (1985).

[11] J. Li, R.-L. Chu, J. K. Jain, and S.-Q. Shen, Phys. Rev. Lett. 102, 136806 (2009).

[12] C. W. Groth, M. Wimmer, A. R. Akhmerov, J. Tworzydło, and C. W. J. Beenakker, Phys. Rev. Lett. 103, 196805 (2009).
[13] H.-M. Guo, G. Rosenberg, G. Refael, and M. Franz, Phys. Rev. Lett. 105, 216601 (2010).

[14] J. Kondo, Prog. Theor. Phys. 32, 37 (1964).

[15] A. C. Hewson, The Kondo Problem to Heavy Fermions (Cambridge University Press, Cambridge, 1997).

[16] I. Adagideli, P. M. Goldbart, A. Shnirman, and A. Yazdani, Phys. Rev. Lett. 83, 5571 (1999).

[17] D. Noid, M. Koszykowski, and R. Marcus, J. Chem. Phys. 71, 2864 (1979).

[18] D. A. Stone, C. A. Downing, and M. E. Portnoi, Phys. Rev. B 86, 075464 (2012).

[19] P. Carmier and D. Ullmo, Phys. Rev. B 77, 245413 (2008).

[20] J. Knoll and R. Schaeffer, Ann. Phys. (N.Y.) 97, 307 (1976).

[21] A. V. Balatsky, I. Vekhter, and J.-X. Zhu, Rev. Mod. Phys. 78, 373 (2006).

[22] M. Srednicki, Quantum Field Theory (Cambridge University Press, Cambridge, 2007).

[23] C. L. Kane and E. J. Mele, Phys. Rev. Lett. 95, 226801 (2005).

[24] C. L. Kane and E. J. Mele, Phys. Rev. Lett. 95, 146802 (2005). 